\title{
Statistical Study of the Correlation Between Topology and Wavelength Usage in Optical Networks with and without Conversion
}

\author{
Christian Fenger $^{1}$, Emmanuel Limal ${ }^{2}$, Ulrik Gliese ${ }^{2}$, and Cathal J. Mahon ${ }^{1}$ \\ 1 Tele Danmark, Telegade 2, DK-2630 Taastrup, Denmark \\ \{cfeng, cmah\}@tdk.dk \\ 2 Research Center COM, Technical University of Denmark, DK-2800 Lyngby, \\ Denmark \\ $\{e l, u g\} @ c o m . d t u . d k$
}

\begin{abstract}
We present a statistical study of wavelength usage in relation to topology characteristics for optical WDM networks with static traffic requirements, where the traffic is routed both with and without wavelength conversion. We identify new general correlations between parameters describing network topologies and wavelength usage. We find that the regularity of a network and the number of spanning trees in a network are accurate and reliable measures of the routing efficiency in terms of wavelengths used to accommodate the required traffic. An empirical formula is given for the average number of wavelengths required to accommodate the traffic as a function of the number spanning trees in the network. We observe that in most cases, the wavelength usage with and without wavelength converters are identical. Consequently, the correlations between network topology and traffic assignment efficiency are true for both types of networks.
\end{abstract}

\section{Introduction}

Optical communication networking is a rapidly growing business. All over the world, many new carriers are investing to deploy completely new networks based on the newest optical fiber technologies. Due to the need for higher bandwidth, the trend moves towards all-optical networks where knowledge of wavelength routed networks becomes increasingly important. Despite this increasingly competitive situation, a general knowledge base on which to build the design and planning of optical networks is still somewhat limited. Up until now, studies of the correlation between network topologies and efficiency in accommodating a certain traffic demand have been based on specific network configurations instead of systematic surveys [1-4]. In this new work, we randomly generate a large number of networks of different topologies and study statistically how well the traffic is accommodated in networks with and without wavelength converters as a function of a number of well defined parameters describing the network topology. We can thereby generate a general knowledge base for network designers.

G. Pujolle et al. (Eds.): NETWORKING 2000, LNCS 1815, pp. 168-175 2000.

(C) Springer-Verlag Berlin Heidelberg 2000 
In the next section we define the problem and describe the routing and wavelength assignment algorithms used for networks with and without wavelength converters. In Section 3 we present the results for networks without wavelength converters. In Section 4 we show and discuss results for networks with wavelength converters and compare these with the results from Section 3.

\section{Problem and Algorithm Description}

The goal of this work is to obtain a general understanding of how different WDM network topologies affect the number of wavelengths required to accommodate a given traffic demand. This is achieved by randomly generating a large number of networks (several million) for given numbers of nodes and links. The wavelength usage is evaluated for each network as a function of the average and variance of the node degree and the number of spanning trees as described in detail in Section 3. The degree, $d$, of a nod e is the number of links incident on the node, as shown in Figure 1, General statistical results are then obtained by averaging over all generated networks for each of these parameters. For each topology we

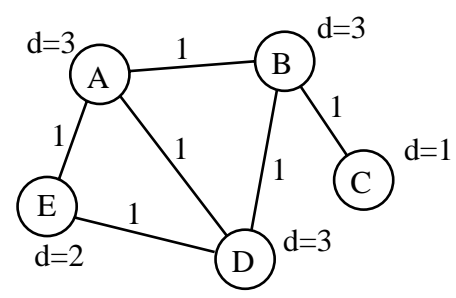

$$
T=\left[\begin{array}{lllll}
0 & 1 & 1 & 1 & 1 \\
1 & 0 & 1 & 1 & 1 \\
1 & 1 & 0 & 1 & 1 \\
1 & 1 & 1 & 0 & 1 \\
1 & 1 & 1 & 1 & 0
\end{array}\right] \quad W=\left[\begin{array}{lllll}
0 & 1 & 2 & 1 & 1 \\
1 & 0 & 1 & 1 & 2 \\
2 & 1 & 0 & 2 & 3 \\
1 & 1 & 2 & 0 & 1 \\
1 & 2 & 3 & 1 & 0
\end{array}\right]
$$

Fig. 1. Example of a network with 5 nodes and 6 links. The weight is one between physical connections. The degree is given for each node. The average node degree is 2.4 , and the variance of the node degree is 0.8 . The network traffic, $T$, and link weight, $W$, matrices are also shown.

assume one fiber per link, no upper limit on the number of wavelengths, uniform traffic, uniform link weight, and bi-directional links. The element of the traffic matrix, $T(i, j)$, gives the traffic between node $i$ and node $j$ in terms of number of wavelengths while the element of the link weight matrix, $W(i, j)$, indicates the number of hops in going from node $i$ to node $j$. As an example, consider the network in Figure 1 for which the weight matrix is determined by using the shortest path connecting each node pair.

\subsection{The Algorithm for Networks Without Wavelength Converters}

The algorithm for networks without wavelength converters contains basically three steps as described below. Due to the huge number of random networks 
generated (several million), we have carefully chosen well-known and efficient algorithms of low complexity. The routing algorithm is an algorithm by Floyd [5] that calculates the shortest path of all node pairs with complexity $\mathcal{O}\left(N^{3}\right)$, where $N$ is the number of nodes in the network. When all routes are known, the wavelength assignment problem is modified into a graph colouring problem. It is done by creating a graph for the path collisions such that each node of this graph corresponds to a path and two nodes are adjacent if their corresponding paths share at least one link in the network topology. The obtained path collison graph must then be coloured such that no two adjacent nodes have the same colour. We chose to solve this problem by using a well-known graph colouring heuristic algorithm called the largest-first or the descending-order-of-degree algorithm 6]. Since the path collision graph has $N(N-1) / 2$ nodes, where $N$ is the number of nodes in the network, then complexity of the largest-first algorithm is $\mathcal{O}\left(N^{4}\right)$. This algorithm basically allocates the lowest number ed colour to the nodes of larger degree first. To ensure that the results for wavelength usage for networks without wavelength converters do not depend on the graph colouring algorithm efficiency, the largest-first algorithm has been compared to the more complex, slower by generally better performing, Dsatur graph colouring algorithm [7. Results are discussed in section 3.

The steps in the algorithm for no wavelength conversion are:

Step 1: A connected network is randomly generated for a given number of nodes and links.

Step 2: The paths between all node pairs are determined using a shortest path algorithm [5].

Step 3: Wavelengths are assigned by modifying the wavelength assignment problem into the graph colouring problem under the restriction that two identical wavelengths cannot share the same link and no wavelength conversion can take place. The graph is coloured using a graph colouring algorithm.

\subsection{The Algorithm for Networks with Wavelength Converters}

For networks with wavelength converters, the algorithm simply contains step 1 and 2 of the algorithm presented in the previous section as no constraints are placed on the wavelength assignment. The wavelengths are literally chosen on a link to link basis.

\section{Results and Discussion for No Conversion}

Efficient network planning is a complex challenge and knowledge of any general trends in network performance in relation to network topology is desirable as it can reduce planning complexity. Therefore, network planners need reliable parameters to characterize network topologies.

The variance of the node degree is one such parameter as it gives a simple measure of the network regularity (i.e. the variance of the node degree) and 


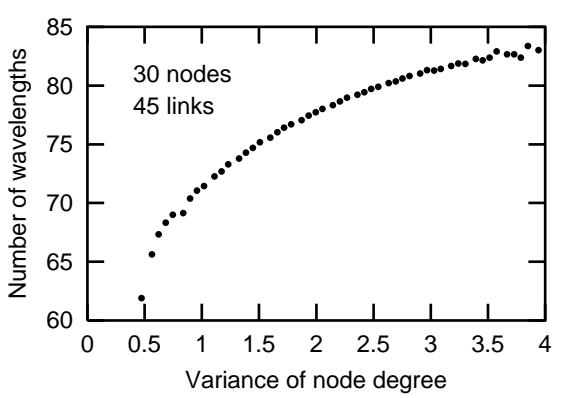

(a)

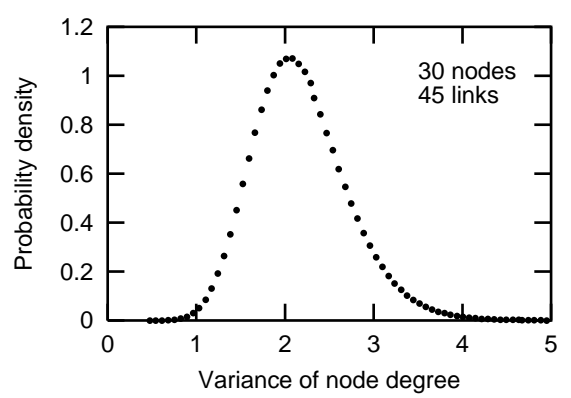

(b)

Fig. 2. (a) Average usage of wavelengths as a function of the variance of the node degree. (b) Probability density of the variance of the node degree. Notice that the distribution is discrete, due to the limited number of possible variances of node degree. Data from 4 million networks are used for the plots.

complements the information given by the average node degree. In Figure 2(a) we have plotted the average number of wavelengths required to accommodate the traffic demand as a function of the variance of the node degree. As seen, the number of wavelengths used grows with the variance, and the network uses a minimum of wavelengths when it is regular (all nodes are of the same degree). To see how many topologies satisfy the low variance requirement, we have also generated the probability density of the variance of the node degree, as shown in Figure 2(b) As seen, very few topologies have very low variance of node degree. This suggests that the number of calculations performed by network optimization algorithms can drastically be reduced.

Another important and more informative parameter for characterizing the topology of an optical network is the number of spanning trees in the network. A tree is a subnetwork of a network, which does not contain any loops, and a spanning tree of a network is a tree containing all nodes of the network. The network shown in Figure 1 contains eight spanning trees. The number of spanning trees is a measure of the number of possible routes between nodes in the network. It easily identifies the restriction in the number of routes due to links that disconnect the network when removed (cut-edges). The calculation of the number of spanning trees, $S$, in a network is easily obtained by calculating the determinant of the product of the matrix $A$ and its transposed matrix $A^{t}$ where $A$ is an incidence matrix of a directed graph obtained by assigning orientations to the edges of the network topology graph such that no self-loops appears with one row deleted [8]:

$$
S=\operatorname{det}\left(A A^{t}\right)
$$

In Figure 3(a) we show the average number of wavelengths required as a function of the number of spanning trees in the network. The simulation points form straight lines for two orders of magnitude, on a double-log scale, indicating a 


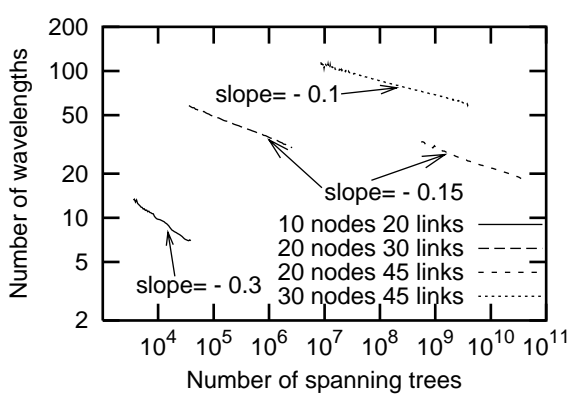

(a)

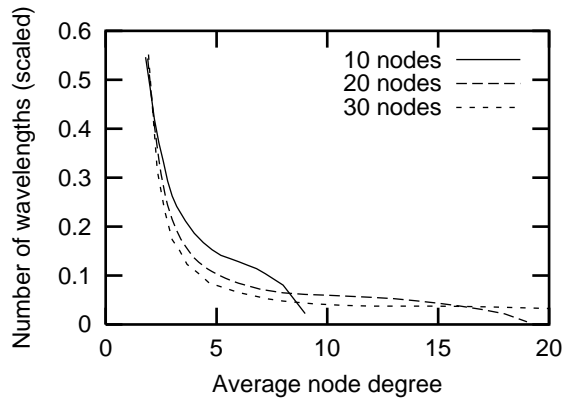

(b)

Fig. 3. (a) Average usage of wavelengths as a function of the number of spanning trees. (b) Average usage of wavelengths, scaled with the total traffic, as a function of the average node degree.

power-law. A comparison of the curves shows that the exponent does not depend on the number of links, but only on the number of nodes, and is approximately given by $-3 / N$.

The equation below gives the dependency of the average wavelength usage, $\langle\lambda\rangle$, on the number of nodes, $N$, the number of links, $L$, and the number of spanning trees, $S$.

For large networks (more than 10 nodes) with a modest average node degree (3-6) we therefore get an empirical law for the average use of wavelengths as function of the number of spanning trees, $S$, when the number of nodes, $N$, and the number of links, $L$, are held constant:

$$
\langle\lambda\rangle=\alpha(N, L) \cdot S^{-3 / N}
$$

Here $\alpha$ is an increasing function of $L$ and a slowly decreasing function of $N$. For very small networks or very strongly or sparsely connected networks the universal feature expressed by the power-law is disturbed. As the number of nodes rises (while the number of links is held constant) $\alpha$ drops, and as the number of links rises (while the number of nodes is held constant) $\alpha$ rises. From this general equation the network planner can get an insight into the wavelength usage of the type of topology to which a specific network belongs.

The average node degree is also a relevant parameter for the network planners. In Figure 3(b) we see the relationship between the average node degree and number of wavelengths required to satisfy the traffic demand. To easily compare the results for different network sizes, the number of wavelengths is scaled with the total traffic demand. It is seen that the gain from raising the average node degree from small values has a significant impact on the decrease in wavelength usage, whereas a raise from larger values only has a small effect. This change in gain from raising the average node degree occurs around an average node degree of 4-5 for all networks, indicating that these are near optimum values. In the 


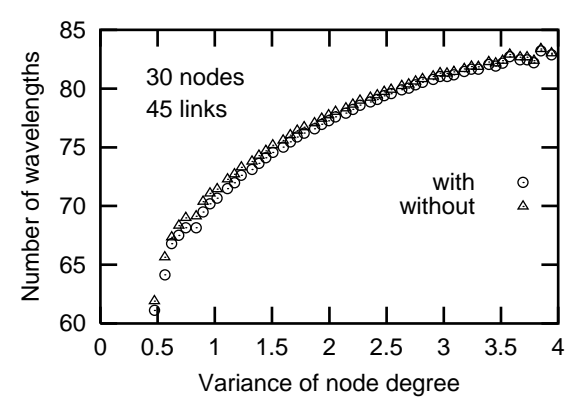

(a)

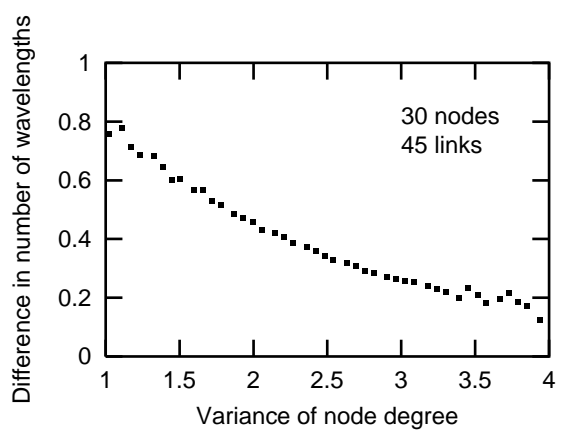

(b)

Fig. 4. (a) Average usage of wavelengths as a function of the variance of the node degree with wavelength converters and without wavelength converters. (b) Difference in average usage of wavelengths between traffic with and without wavelength converters as a function of the variance of the node degree.

following section, we investigate wavelength usage for networks with wavelength converters as a function of the same topology parameters, namely the variance of the node degree and the number of spanning trees. Second it is seen that the performance of the algorithm actually rises with the size of the network. Where the performance is measure as the number wavelengths used scaled with the total traffic when the average node degree is held constant. As the total traffic scales with square of the number of nodes and the number of links for a constant average degree scales with the number of nodes, then the number of wavelengths divided by the total traffic should be constant in a network, which does not scale. Here we see that the traffic is more efficiently accommodated for larger networks.

\section{Comparison with Wavelength Conversion}

The statistical results estimated over millions of networks show that the correlation between topologies and wavelength usage is exactly similar for networks with and without wavelength converters. However, small differences in wavelength usage were noticed and are discussed in this section. In Figure 4(a) the average usage of wavelength is plotted for networks with and without wavelength converters as a function of the variance of node degree for networks of 30 nodes and 45 links. Although it reduces the blocking probability and reduces management complexity for dynamic traffic assignment it has no (or only a very small) effect on static routing. The curves indicate a very small difference in the number of wavelengths required to assign the traffic. The difference, when it exists, is generally of the order of one or two wavelengths which represents, on average for all networks, an increase in wavelength usage of only $0.5 \%$.

Contrary to what was expected, the difference in wavelength usage increases for networks with small variances (regular networks) as shown in Figure 4(b). 
We believe that this is mainly due to the increase in the inefficiency of the largest-first graph colouring algorithm as the networks become more regular. To verify this assertion, we have in a provisional study compared the largestfirst algorithm to the more complex graph colouring algorithm, Dsatur [7], in which the Dsatur algorithm proved better than the largest-first by sometimes using fewer wavelengths in the few cases where the network with wavelength converters had a lower wavelength usage. This matter is, however, still under investigation. See also 9 for a comparison between different heuristic methods of graph colouring.

In Figure 5, the wavelength usage for networks with and without wavelength converters is plotted versus the number of spanning trees of the network topologies. Results for networks without wavelength converters are shown for the largest-first graph colouring algorithm. It is clearly seen that the analytical formula of section 3 is still valid for networks with wavelength converters.

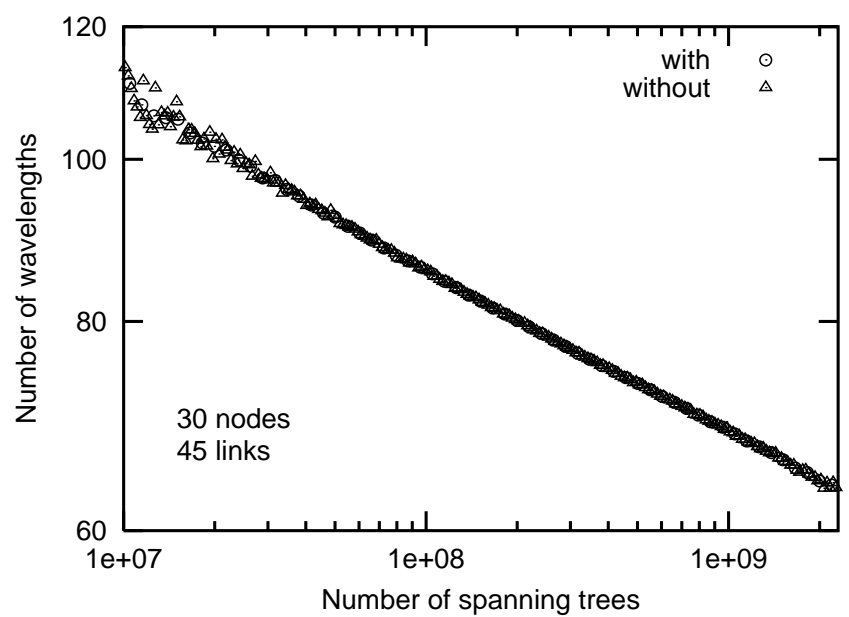

Fig. 5. Average usage of wavelengths as a function of the number of spanning trees with wavelength converters and without wavelength converters.

\section{Conclusions}

This paper contains a thorough investigation of the influence of network topology on wavelength usage. This investigation was done through an exhaustive statistical study of randomly generated topologies for networks with and without wavelength converters. We define general parameters that accurately describe network topology properties and clearly identify the correlation between the topology properties and the wavelength usage in networks. The correlations are exactly identical for networks with and without wavelength converters. It is 
shown that for a given number of nodes there are only very few topologies that perform efficiently in terms of wavelength usage and we indicate the criteria that identifies them. These criteria can be used to simplify the optimization process during network planning. We have shown that, in general, networks that have an average node degree of 4-5 and a low node degree variance are among the best possible in terms of wavelength usage. Furthermore, we have clearly identified the number of spanning tree s as a very accurate measure of the quality of a network in terms of traffic accommodation efficiency, and we have derived a simple equation for the average number of wavelengths required for a given topology as a function of the number of the nodes, links, and spanning trees it contains. In conclusion, we present a statistical analysis of the general network behavior in terms of wavelength usage over a very large number of network topologies and obtain general trends as well as a formula that can drastical ly narrow down the number of solutions that need to be considered when designing a network.

\section{References}

1. Tan, T.K. and Pollard, J.K.: 'Determination of minimum number of wavelengths required for all-optical WDM networks using graph coloring', Electronics Letters, Vol.31, pp.1895-1897, October 1995.

2. Subramaniam, S., Barry, R., 'Wavelength Assignment in Fixed Routing WDM Networks',IEEE Int. Conf. on Comm., Vol.1, pp.406-410, 1997.

3. Mokhtar, A., Aziõglu, M., 'Adaptive Wavelength Routing in All-Optical Networks', IEEE/ACM Trans. Networking, Vol.6, pp.197-206, April 1998.

4. Baroni, S., Bayvel, P., Gibbens, R.J., and Korotky, S.K., 'Analysis and Design of Resilient Multifiber Wavelength-Routed Optical Transport Networks', J. Lightwave Technol., Vol.17, pp.743-758, May 1999.

5. Floyd, R.W.: 'Algorithm 97 Shortest Path', Communications of the ACM, Vol.5, p.345, 1962.

6. Welsh, D.J.A. and Powell, M.B., 'An upper bound for the chromatic number of a graph and its application to timetable problems', The Computer Journal, Vol.10, pp.85-86, 1967.

7. Brelaz, D., 'New Methods to color the vertices of a graph', Communications of the Association for Computing Machinery, pp.251-256, 1979.

8. Thulasiraman, K. and Swamy, M., Graphs: 'Theory and Algorithms', John Wiley ES Sons, 1992.

9. Parkinson, E. and Warren, P.R., 'A comparison of graph colouring techniques', South African Comp. J., Vol.14, pp.14-19, 1995. 